\title{
La protección de la salud de las personas frente al libre comercio de mercancías en la Comunidad Andina
}

\author{
Yovana Reyes Tagle*
}

\section{RESUMEN}

El artículo 73 del Acuerdo de Cartagena reconoce que la protección de la vida y salud de las personas puede justificar la adopción de restricciones a las importaciones en la Comunidad Andina (CAN). Esta investigación analiza la idoneidad que debe darse entre la medida nacional adoptada y el objetivo perseguido. Se usa como caso de estudio las restricciones cuantitativas introducidas para reducir la emisión de gases de efecto invernadero para proteger la salud de las personas por parte de Ecuador y su justificación a la luz del Acuerdo de Cartagena. Se plantea que el análisis de una restricción a la importación de mercancías adoptada para proteger la salud de las personas no solo exige una evaluación cuantitativa de la contribución de dicha restricción para lograr el objetivo deseado, sino, que el sector o actividad que causa el problema sea el más significativo; es decir, un enfoque cualitativo.

Palabras clave: Salud pública, libre comercio, restricciones a las importaciones, libre circulación de mercancías, Comunidad Andina.

The protection of human health against free trade of goods in the Andean Community

\section{Abstract}

Article 73 of the Cartagena Agreement recognizes that the protection of human life and health can be justified through the adoption of import restrictions in the Andean Community. This paper analyzes the suitability between the national measure adopted and the objective pursued. The quantitative restrictions introduced by Ecuador to reduce the emission of greenhouse gases to protect human health and their justification in the light of the Cartagena Agreement are used as a case study. The paper argues that the analysis of an import restriction adopted to protect human health does not only require a quantitative assessment of the

* Doctora en Derecho (LL.D.) y magíster en Derecho (LL.M.) por la Universidad de Helsinki, Finlandia. Abogada, docente y miembro del Comité Directivo de la Maestría de Derecho Internacional Económico de la Pontificia Universidad Católica del Perú. Correo electrónico: yreyes@pucp.edu.pe

iD https://orcid.org/0000-0001-8563-4464 
contribution of the restriction to achieve the desired objective; namely, that the sector or activity that causes the problem is the most significant. A qualitative approach is necessary.

Keywords: Public health, import restrictions, free movement of goods, Andean Community.

\section{La protección de la salud de las personas y la eliminación de restricciones a las importaciones en la Comunidad Andina}

\subsection{La obligación de eliminar las restricciones al comercio y la excepción que permite el Acuerdo de Cartagena para proteger la salud de las personas}

La Constitución de la Organización Mundial de la Salud (OMS) define la salud como «un estado de completo bienestar físico, mental y social, y no solamente la ausencia de afecciones o enfermedades». Este enfoque es consistente con lo que estipula el artículo 12 numeral 1 del Pacto Internacional de Derechos Económicos, Sociales y Culturales (Pidesc), el cual reconoce «el derecho de toda persona al disfrute del más alto nivel posible de salud física y mental». Conforme al artículo 12 numeral 2 literal c) del Pidesc, entre las medidas que deben adoptar los Estados para garantizar este derecho se encuentran aquellas dirigidas a la "prevención y el tratamiento de las enfermedades epidémicas, endémicas, profesionales y de otra índole, y la lucha contra ellas». El artículo 2 numeral 1 del Pidesc precisa que los Estados deben adoptar medidas para hacer efectivos estos derechos. Consistente con este enfoque, el artículo 24 de la Carta Andina para la Promoción y Protección de los Derechos Humanos (no vinculante) reafirma el compromiso de los Países Miembros de dar cumplimiento a las normas del Pidesc. Específicamente, el artículo 24 numeral 7 de la Carta se refiere a su compromiso de adoptar medidas destinadas a lograr el «más alto nivel posible de salud física y mental» en la región andina.

Asimismo, las normas en la CAN obligan a los Países Miembros a eliminar las restricciones injustificadas e innecesarias al comercio, pero reconocen que la protección de la salud humana puede justificar y hacer necesaria la adopción de medidas que restrinjan el comercio andino. El artículo 72 del Acuerdo de Cartagena consagra el principio de libre circulación de mercancías y estipula que «el Programa de Liberación tiene por objeto eliminar los gravámenes y las restricciones de todo orden que incidan sobre la importación de productos originarios del territorio de cualquier País Miembro». Esta obligación es complementada por el artículo 73 del Acuerdo de Cartagena que define el concepto de «restricciones de todo orden» como «cualquier medida de carácter administrativo, financiero o cambiario, mediante la cual un País Miembro impida o dificulte las importaciones, por decisión unilateral». 
El artículo 73 del Acuerdo de Cartagena enumera las excepciones al principio de libre circulación de mercancías, entre ellas: la protección de la vida y salud de las personas, los animales y los vegetales. Estas excepciones permiten a los Países Miembros justificar la adopción de medidas para proteger el interés público nacional, otorgándoles un espacio para definir sus políticas de protección frente a la estricta obligación de eliminar las restricciones a las importaciones. Como sostienen Armingeon, Milewicz, Peter y Peters (2011, p. 87), este tipo de cláusulas reflejan el reclamo de prioridad por la soberanía de los Estados. El artículo XX del Acuerdo General sobre Aranceles Aduaneros y Comercio (GATT) contiene una excepción similar. De esta manera, las excepciones generales contempladas en esta norma multilateral son comúnmente utilizadas en los acuerdos comerciales internacionales para salvaguardar la autonomía regulatoria de los Estados. Su uso ha aumentado también en los acuerdos internacionales de inversiones (Mitchell y Sheargold, 2015, p. 150).

Asimismo, la CAN tiene otras normas que tratan el tema de las restricciones al comercio y la protección a la salud humana. Por ejemplo, el artículo 12 de la Decisión 515 que regula el Sistema Andino de Sanidad Agropecuaria permite que los Países Miembros, la Comisión y la Secretaría General de la CAN (SGCAN) adopten las normas sanitarias y fitosanitarias que sean necesarias para «contribuir al mejoramiento de la salud y la vida humana». El artículo 4 de la Decisión 827 incorpora a la protección de la salud o seguridad humana, de la vida o la salud animal o vegetal como parte de la definición de objetivos legítimos que los Países Miembros pueden buscan alcanzar mediante la adopción de un reglamento técnico.

Howse (2012, p. 452) ha señalado que las normas de la Organización Mundial del Comercio (OMC) representan un balance adecuado de valores entre los objetivos de política pública que podrían estar en tensión con la liberalización del comercio a la cual se le ha dado una considerable importancia. Las normas andinas muestran también ese balance de valores entre, por un lado, el principio de libre circulación de mercancías y, por otro lado, los objetivos de política pública.

En atención a lo dispuesto en el artículo 4 de la Decisión 515 y el artículo 10 inciso 1 de la Decisión 827, se puede determinar que las normas andinas consideran que la protección de la vida y salud de las personas se refiere a la necesidad de prevenir o impedir que existan riesgos de difusión de plagas o enfermedades. Es ilustrativo señalar que en la OMC el concepto de protección a la vida y salud de las personas también ha sido debatido en el marco del GATT. Al respecto, en el caso Unión Europea-Focas, el órgano de apelación señaló que:

Es posible que la protección de la salud y la vida de las personas y de los animales o la preservación de los vegetales impliquen una atención particular a la protección de 
o contra determinados peligros o riesgos. Por ejemplo, los conceptos de «riesgo»y "protección» están recogidos expresamente en el Acuerdo MSF, que elabora normas para la aplicación del apartado b) del artículo XX. (OMC, 22 de mayo de 2014, párr. 5.197)

Las normas andinas revisadas coinciden con el alcance del concepto de protección de la vida y salud de las personas expresado por el Órgano de Apelación en el caso Unión Europea-Focas. El artículo 73 del Acuerdo de Cartagena responde a la necesidad de los Países Miembros de proteger la vida y salud de las personas frente a cualquier riesgo o peligro, y es por esta razón que permite la adopción de restricciones al comercio. La norma no se limita a proteger la salud física, se incluye la protección de la salud mental coincidiendo con el reconocimiento que realiza el Pidesc, el concepto de salud de la OMS y la Carta Andina para la Promoción y Protección de los Derechos Humanos.

\subsection{La aplicación de restricciones cuantitativas para reducir la emisión de gases de efecto invernadero en Ecuador: la protección de la salud de las personas frente al principio de libre circulación de mercancías en la Comunidad Andina}

La emisión de gases de efecto invernadero (GEI) resulta perjudicial para la salud humana. Los resultados de las investigaciones así lo demuestran. Uno de los retos para los Estados es adoptar medidas para prevenir las enfermedades que esta emisión ocasiona. La OMS ha destacado la importancia de reducir la emisión de GEI y su relación con la salud humana. Así, esta reducción «mediante mejoras del transporte y de las elecciones en materia de alimentos y uso de la energía pueden traducirse en mejoras de la salud, en particular a través de la reducción de la contaminación atmosférica» (OMS, 1 de febrero de 2018). Asimismo, la OMS señala que «El cambio climático influye en los determinantes sociales y medioambientales de la salud, a saber, un aire limpio, agua potable, alimentos suficientes y una vivienda segura» (OMS, 1 de febrero de 2018). Los cálculos aproximados de los costos que acarrea el daño causado para la salud son reveladores: «Se estima que el coste de los daños directos para la salud (es decir, excluyendo los costes en los sectores determinantes para la salud, como la agricultura y el agua y el saneamiento) se sitúa entre 2000 y 4000 millones de dólares de aquí a 2030» (OMS, 1 de febrero de 2018).

Las cifras con respecto al daño ocasionado en la vida y salud de las personas son preocupantes. Se ha previsto que «entre 2030 y 2050 el cambio climático causará unas 250.000 defunciones adicionales cada año, debido a la malnutrición, el paludismo, la diarrea y el estrés calórico" (OMS, 1 de febrero de 2018). Se calcula que esas defunciones se darían en el siguiente rango: «38.000 por exposición de personas ancianas al calor; 48.000 por diarrea; 60.000 por paludismo; y 95.000 por desnutrición infantil»(OMS, 1 de febrero de 2018). 
Los Estados pueden adoptar medidas para lidiar con este problema. A fin de contribuir con la reducción de GEI, Ecuador adoptó las resoluciones 65 y 66 del Comité de Comercio Exterior (Comex) en junio de 2012, estableciendo restricciones al comercio, tales como aranceles y restricciones cuantitativas. Este trabajo analiza las segundas.

Mediante las resoluciones 65 y 66 del Comex, Ecuador impuso una restricción cuantitativa de importación de CKD de vehículos (determinada en función de unidades de CKD y por valor). De esta manera, se establecía una restricción cuantitativa anual de importación para vehículos clasificados en 16 subpartidas arancelarias «fijada por unidad de vehículos y por valor, así como la autorización a treinta y ocho (38) importadores de poder ingresar vehículos al Ecuador» (SGCAN, 18 de abril de 2013, pp. 2-3).

Sin embargo, al surgir el tema de la violación del principio de libre circulación de mercancías reconocido por el Acuerdo de Cartagena, Ecuador alegó que la medida se encontraba justificada porque buscaba proteger la salud de las personas mediante la reducción de la emisión de GEI (SGCAN, 18 de abril de 2013, pp. 6-7). A fin de justificar la adopción de restricciones cuantitativas, Ecuador se amparó en el artículo 73 literal d) del Acuerdo de Cartagena que permite de manera excepcional introducir restricciones al comercio para proteger la vida y salud de las personas.

Las restricciones cuantitativas se refieren a medidas que limitan la cantidad de importaciones o exportaciones de un producto. Esta limitación no requiere ser demostrada mediante la medición de los efectos de una medida nacional, sino a través del análisis del diseño, construcción y estructura de dicha medida (Van den Bossche y Prévost, 2016, p. 62; OMC, 22 de diciembre de 2016, párr. 8.1.b). Sin duda, las restricciones cuantitativas son barreras de acceso al mercado prohibidas. En el caso de la medida ecuatoriana, no se discute si la medida constituye o no una restricción al comercio contraria al artículo 72 del Acuerdo de Cartagena. Las restricciones cuantitativas se encuentran dentro del alcance del concepto de restricciones de todo orden de esta norma. Al respecto, las Resoluciones de la SGCAN apuntan a que este tipo de restricciones cuantitativas violan el principio de libre circulación de mercancías (SGCAN, 18 de abril de 2013, pp. 7-8, 12). Las interpretaciones de los Grupos Especiales y el Órgano de Apelación en la OMC también son claras con respecto a la prohibición de introducir restricciones cuantitativas (OMC, 31 de mayo de 1999, párr. 9.63).

En consecuencia, para poder introducir y mantener la aplicación de las restricciones cuantitativas con el fin de proteger la salud de las personas es necesario justificarlas al amparo de la excepción permitida por el artículo 73 del Acuerdo de Cartagena. Conforme lo ha planteado el Tribunal de Justicia de la CAN (TJCAN) y la SGCAN, esto hace necesario evaluar las condiciones que se requieren cumplir para aceptar tales restricciones. 
2. La justificación de la adopción de una restricción al comercio a la luz del Acuerdo de Cartagena según la jurisprudencia del Tribunal de Justicia de la Comunidad Andina

\subsection{Las condiciones para justificar la adopción de restricciones al comercio en el marco de la Comunidad Andina}

En la OMC, el artículo XI del GATT requiere que los Miembros eliminen las restricciones cuantitativas a las importaciones. Asimismo, el artículo XX del GATT permite que los Miembros adopten restricciones al comercio contrarias al artículo XI del GATT de manera excepcional. Una de esas excepciones es la protección de la vida y salud de las personas. El preámbulo del artículo XX del GATT impone condiciones para la aplicación de esta excepción y exige que estas restricciones al comercio no deban constituir una discriminación arbitraria o injustificada o una restricción encubierta del comercio. Las decisiones en la OMC han demostrado la dificultad para superar los altos estándares establecidos por este preámbulo (Wells, 2014, p. 228; Kanade, 2012, p. 409). No obstante, el concepto de salud y la vida de las personas del artículo XX literal b) del GATT está abierto a la amplia interpretación. Este concepto podría incluir medidas que protejan todos los aspectos de la salud física y psicológica y el bienestar (Harris y Moon, 2015, p. 22).

El Acuerdo sobre Obstáculos Técnicos al Comercio (Acuerdo OTC) reconoce el derecho de los Estados a aplicar reglamentos técnicos para proteger sus objetivos legítimos, entre ellos, la protección de la vida y salud de las personas. Sin embargo, el ejercicio de este derecho o la autonomía de los Estados están condicionados a fin de evitar que se apliquen obstáculos innecesarios al comercio (Artículo 2.2 del Acuerdo OTC). En el caso Comunidades Europeas - Sardinas, el Grupo Especial consideró el tema del límite de la autonomía de los Miembros con relación a la adopción de los reglamentos técnicos. Al referirse al alcance del artículo 2 párrafo 2 del Acuerdo OTC, el Grupo Especial sostuvo que esta norma se caracteriza porque impone condiciones a los Estados, como la no discriminación arbitraria e injustificada (OMC, 29 de mayo de 2002, párr. 7.120).

En esta misma línea, el Acuerdo sobre la Aplicación de Medidas Sanitarias y Fitosanitarias (Acuerdo MSF) otorga a los Estados la facultad de adoptar medidas sanitarias y fitosanitarias siempre que cumplan con las obligaciones previstas en el Acuerdo. Aun cuando lo que se busca es proteger la vida y salud de las personas, el Acuerdo MSF busca garantizar que las medidas nacionales sean justificadas, tengan como sustento principios científicos y no constituyan una restricción encubierta del comercio (Artículo 2.2 y 2.3 del Acuerdo MSF). 
En la Unión Europea, el artículo 34 del Tratado de Funcionamiento de la Unión Europea (TFUE) consagra el principio de libre circulación de mercancías y obliga a los Estados miembros a eliminar las restricciones cuantitativas y las medidas de efecto equivalente a las restricciones cuantitativas. El artículo 36 del TFUE contiene las excepciones a este principio, tales como la protección de la vida y salud de las personas. Además, esta disposición exige el cumplimiento de algunos requisitos para que los Estados puedan introducir una restricción al comercio contraria a este principio, es decir, «tales prohibiciones o restricciones no deberán constituir un medio de discriminación arbitraria ni una restricción encubierta del comercio entre los Estados miembros.» El lenguaje de esta disposición se asemeja al artículo XX del GATT.

Por otro lado, existen acuerdos comerciales regionales que optan por un enfoque diferente al del GATT y el TFUE en lo que se refiere a la regulación de las excepciones a la obligación de los Estados de eliminar las restricciones al comercio. En el Mercosur, el artículo 1 del Tratado de Asunción de 1991 contempla la eliminación de las «restricciones no arancelarias a la circulación de mercaderías y de cualquier otra medida equivalente». El artículo 2 del Anexo I de este Tratado se remite al artículo 50 del Tratado de Montevideo de 1980 y excluye del concepto de restricciones a las medidas unilaterales adoptadas al amparo de las excepciones previstas en esta norma. El Tratado de Montevideo establece como excepciones, entre otras, la protección de la vida y salud de las personas, los animales y los vegetales. Sin embargo, estas normas no introducen condiciones para su aplicación. Por ello, el Tribunal Permanente de Revisión (TPR) del Mercosur ha señalado que no existe un marco legal que señale de manera clara y concreta los criterios necesarios para analizar estas excepciones (TPR del Mercosur, 2005, párr. 10). El TPR ha propuesto cuatro criterios para analizar las excepciones, recurriendo a la jurisprudencia del Tribunal de Justicia de la Unión Europea (TJUE) (Reyes Tagle, 2013, pp. 7-8).

De manera similar a lo regulado en el Mercosur, el artículo 73 del Acuerdo de Cartagena enumera las excepciones al principio de libre circulación de mercancías sin imponerse condiciones explícitas para su utilización, salvo el requisito de que estén «destinadas a» la protección de la salud humana. Aparentemente, los Estados tienen una considerable discreción.

Si se compara la redacción del artículo XX b) del GATT con el artículo 73 literal d) del Acuerdo de Cartagena existen diferencias notables. En el GATT se incorpora la prueba de la necesidad y se exige el cumplimento de tres condiciones para aceptar una restricción al comercio por motivos de salud pública. El Acuerdo de Cartagena, al igual que en el Tratado de Montevideo de 1980, no introduce ninguna de esas condiciones del artículo XX del GATT o las del artículo 36 del TFUE. Al respecto, ha sido el TJCAN quien ha interpretado el artículo 73 del Acuerdo de Cartagena e 
introducido algunas condiciones para que la medida adoptada por un País Miembro pueda ser justificada como una excepción al principio de libre circulación de mercancías que no vaya más allá de lo necesario.

A partir de su sentencia en el Proceso 3-AI-96, (TJCAN, 29 de abril de 1997, p. 17) y reiterada en 2017 en el Proceso 2-AN-2015, el TJCAN ha precisado que para que una restricción al comercio pueda justificarse, es necesario que se cumplan con las siguientes condiciones:

Debe ser idónea para cumplir la finalidad legítima perseguida (una finalidad legítima sería la protección de la vida y salud de las personas, los animales y vegetales); es decir, debe ser causa directa e inmediata para la solución del problema detectado. Así, si el problema son los GEI, debe analizarse si la medida restrictiva al comercio subregional andino constituye o no una causa directa e inmediata para mitigar la emisión de GEI. No debe haber otra medida que, sin obstaculizar el comercio, podría cumplir el mismo objetivo perseguido (la finalidad legítima) por la medida materia de análisis.

La medida no debe ser discriminatoria. (TJCAN, 2 de mayo de 2017, p. 27)

El TJCAN ha recurrido al artículo 30 del Tratado de la Comunidad Económica Europea (actual artículo 34 del TFUE) para llenar el vacío dejado por el Acuerdo de Cartagena e introducir las condiciones que deben cumplir los Países Miembros. Concretamente, el TJCAN ha considerado al TFUE y a las normas de la OMC sobre restricciones cuantitativas como "valiosa fuente supletoria del derecho» (TJCAN, 2 de mayo de 2017, p. 33; TJCAN, 29 de abril de 1997, p. 17; TJCAN, 30 de noviembre de 2000, p. 14). La SGCAN también ha aplicado estos criterios desarrollados por el TJCAN (SGCAN, 18 de abril de 2013, p. 10).

A partir del caso Ecuador y la emisión de GEI, este trabajo analiza uno de estos criterios para aceptar una restricción al comercio justificada: la idoneidad de la medida, es decir, la relación de causalidad entre la medida adoptada y el objetivo que se busca alcanzar. El TJCAN ha reiteradamente sostenido que existe la necesidad de que exista una vinculación directa e inmediata entre la medida y el problema presentado (TJCAN, 7 de agosto de 1998, p. 24; TJCAN, 9 de marzo de 1998, p. 8).

\subsection{La relación de causalidad entre las medidas restrictivas del comercio adoptadas por Ecuador y el objetivo de política buscado a la luz del Acuerdo de Cartagena}

La SGCAN conceptualiza el requisito de causalidad y sostiene que, para aceptar una excepción al principio de libre circulación de mercancías, la medida adoptada por el País Miembro «debe estar vinculada directa e inmediatamente con la solución al problema específico» (SGCAN, 18 de noviembre de 2013, p. 8; SGCAN, 8 de febrero de 2005, p. 4.24). Al analizar la causalidad entre las medidas adoptadas por Ecuador y el objetivo de política buscado, la SGCAN concluyó que: 
[...] el sector transporte tiene una contribución poco significativa en la emisión de GEI (3\%), existiendo otros sectores y actividades que coadyuvan a una mayor generación de este tipo de emisiones, de lo cual se determina que la importación de vehículos y materiales $\mathrm{CKD}$ no podría ser calificada como la causa directa de riesgos a la salud por efecto de estas emisiones, que justifique la imposición de medidas restrictivas como las contenidas en las Resoluciones 65 y 66 del COMEX. (SGCAN, 18 de abril de 2013, p. 15)

El TJCAN concluyó que este análisis de la SGCAN con respecto a la causalidad de la medida era un "absurdo» (TJCAN, 2 de mayo de 2017, p. 33). La SGCAN evaluó la contribución de la medida con el logro del objetivo buscado teniendo en cuenta el porcentaje en la emisión de GEI ocasionado por el sector transporte. A partir de esta constatación, la SGCAN determinó que al ser ese porcentaje bajo, no se cumplía con la relación de causalidad. En otras palabras, la SGCAN rechazó la idoneidad de la medida por considerar muy débil la relación entre dicha medida y el objetivo perseguido. Como resultado, la protección de la salud humana se descuidó en comparación con la liberalización del comercio. Esta posición de la SGCAN es cuestionable por varias razones. Primero, la aplicación de las excepciones al principio de libre circulación de mercancías no exige que la actividad que genera un riesgo para la salud humana sea la más significativa. Segundo, la existencia de otros sectores que contribuyen en la emisión de GEI no excluye la posibilidad de adoptar medidas que busquen reducir o eliminar el efecto negativo contra la salud humana causado por uno de esos sectores ni exige que se tenga que tomar medidas primero con respecto al sector más significativo.

Tercero, el sector transporte es una de las causas de la emisión de GEI; por lo tanto, aplicando la definición de causalidad planteada antes por la propia SGCAN, la medida adoptada por Ecuador está vinculada directa e inmediatamente con la reducción de dicha emisión. Cualquiera que sea el grado de contribución de un sector o actividad a la emisión de GEI debe ser considerada como causa directa de riesgos a la salud humana y, por ende, se cumple con el requisito de la idoneidad para aplicar una medida restrictiva del comercio. Al referirse a la «solución al problema específico» nada indica que la medida tenga que solucionar todo el problema o una parte substancial del problema.

Cuarto, la SGCAN reconoce que el sector transporte contribuye a la emisión de GEI. Sin embargo, al considerar que existen otros factores o actividades que tienen una mayor contribución en dicha emisión, la SGCAN descarta la posibilidad de considerar a este sector como «la causa directa de riesgos a la salud por efecto de estas emisiones». Es decir, la SGCAN entendería que causa directa implica que se contribuya de manera significativa al problema específico. No obstante, aun cuando el sector transporte tenga una mínima contribución en la emisión de GEI comparado con otros sectores 
o actividades, sigue siendo una de las causas de y, por lo tanto, se «vincula con» dicha emisión de manera directa e inmediata. Esa vinculación no se descarta realizando una cuantificación de dicha contribución como lo hizo la SGCAN.

Quinto, la reducción de las emisiones del transporte que incluye el carbono negro, el ozono troposférico y los precursores de aerosol puede tener beneficios para la salud humana y la mitigación a corto plazo (IPCC, 2014, p. 22). La SGCAN cuantificó la contribución del sector transporte en la emisión de GEI, introduciendo con esto un requisito adicional. En contraste, no tuvo en cuenta, en términos cuantitativos, la reducción de la emisión de GEI que resultaría como resultado de la aplicación de la restricción cuantitativa en el corto o largo plazo. Sin un examen de la magnitud de dicha contribución sostenida con datos estadísticos es difícil aceptar una decisión de la SGCAN que descarta el cumplimiento del requisito de la causalidad porque el sector transporte solo contribuye con un 3\%. Un enfoque integral requiere que el análisis se centre no solo en el impacto de la medida en el comercio sino también en el objetivo no comercial protegido que se busca alcanzar.

En el análisis de los requisitos para aceptar una excepción al comercio, no se puede perder de vista el grado de importancia de los objetivos que se buscan lograr, en este caso, la salud humana. Este criterio ha sido desarrollado por el el Órgano de Apelación en el caso Brasil-Neumáticos en el cual concluyó que «la contribución de la medida debe confrontarse con el grado en que restringe el comercio, teniendo en cuenta la importancia de los intereses o los valores a que responde el objetivo perseguido con ella» (OMC, 3 de diciembre de 2007, párr. 210). El razonamiento de la SGCAN contradice el reconocimiento de la facultad que tienen los Estados para definir sus políticas públicas y el grado de protección de sus objetivos legítimos. Las normas andinas y de la OMC no limitan esta facultad de los Estados.

De aceptarse la posición de la SGCAN adoptada en el caso de Ecuador se llegaría a la conclusión cuestionable de que los Estados no pueden adoptar medidas para lidiar con determinado problema a menos que primero se adopte medidas que vayan dirigidas a contrarrestar los principales factores causantes del mismo. Ninguna de las normas andinas revisadas apoya tal entendimiento de la SGCAN. Asimismo, en la $\mathrm{OMC}$ se ha prestado atención al grado de contribución de la medida con el objetivo perseguido. En los casos República Dominicana-Cigarrillos y Brasil-Neumáticos, la mayor parte del razonamiento de los Grupos Especiales se enfocó en analizar esta contribución. La consideración del valor de la medida y su efecto restrictivo fue limitada en sus decisiones (Kapterian, 2010, p. 123).

Según la jurisprudencia del TJCAN y las Resoluciones de la SGCAN, una medida que potencialmente puede afectar las importaciones, por más mínima que sea dicha 
afectación, puede constituir una restricción al comercio contraria a los artículos 72 y 73 del Acuerdo de Cartagena. En este sentido, el TJCAN ha sostenido que es «suficiente que tenga como efecto directo o indirecto el de dificultar la libre circulación de mercancías» (TJCAN, 2 de agosto de 2000, p. 12).

La SGCAN destaca que «con la finalidad de garantizar el adecuado funcionamiento del mercado ampliado es menester pronunciarse respecto de los casos que directa o indirectamente, actual o potencialmente, puedan restringir el comercio subregional mediante actos que pudieran tener efectos limitantes del comercio" (SGCAN, 18 de junio de 1999, pp. 5-7). También, si una restricción a las importaciones tiene una corta duración puede ser considerada como una restricción incompatible con el Acuerdo de Cartagena. Las medidas nacionales que limitan el acceso al mercado de productos originarios de un País Miembro, como las licencias previas, son contrarias a los artículos 72 y 73 del Acuerdo de Cartagena (SGCAN, 8 de mayo de 2003, p. 5). Esto es relevante para efectos de calificar una medida nacional como restricción al comercio, ya que no se tendrá en cuenta cuánto ha durado la aplicación de la medida. Por lo cual, aun cuando la medida se encuentre vigente por solo unos meses, y no sea discriminatoria puede ser considerada como una restricción al comercio (SGCAN, 10 de setiembre de 1999, pp. 5-6). Por ende, no hay una regla de minimis para calificar las restricciones al comercio como ilegales. En claro contraste, la SGCAN adoptó una regla de minimis para aceptar la aplicación de una restricción al comercio por motivos de salud humana en el caso de Ecuador.

Si una medida nacional potencial, de corta duración y de mínimo impacto en las importaciones constituye una violación del Acuerdo de Cartagena, no puede aceptarse el argumento de la SGCAN de que por el hecho de que el sector transporte en Ecuador solo contribuye en un 3\% en la emisión de GEI la medida no es idónea. La calificación de una medida nacional como restricción al comercio, que refleja claramente la defensa estricta del principio de libre circulación de mercancías, contrasta con el enfoque restringido adoptado por la SGCAN al momento de evaluar la protección de la salud humana. Esta diferencia de enfoque adquiere mayor relevancia cuando lo que se busca es proteger derechos fundamentales.

En la OMC, en los casos China-Materias primas e Indonesia-Carne de pollo, los Grupos Especiales coincidieron en señalar que «La mera posibilidad de que se limite el comercio es suficiente para que haya restricción» (OMC, 22 de febrero de 2012, párr. 7.1081; OMC, 17 de octubre de 2017, párr. 60). Este enfoque se asemeja a la forma cómo la SGCAN califica las medidas nacionales como restricción al comercio. Sin embargo, esto no ha impedido que en la OMC consideren la relevancia de proteger la vida y salud de las personas. 
Si bien el TJCAN ha entendido que la interpretación de las excepciones debe realizarse de manera restringida (TJCAN, 2 de mayo de 2017, p. 33; TJCAN, 20 de agosto de 1999, p. 10; TJCAN, 3 de junio de 2015, p. 16), esta interpretación no implica introducir exigencias no razonables que limitan la capacidad de los Estados para proteger intereses de política pública, específicamente, derechos humanos que tienen la obligación de proteger. Con respecto a los derechos humanos, el TJCAN ha recibido algunas críticas. Entre otras razones, Rincón Eizaga (2013) sostiene que este tribunal:

[...] se ha caracterizado por una gran pasividad en esta materia, no solo reflejada en la ausencia de jurisprudencia en materia de protección de los derechos humanos en el ámbito comunitario, sino también en su rechazo a participar en la génesis y elaboración de la Carta Andina para la Promoción y Protección de los Derechos Humanos (p. 427).

No obstante, el enfoque adoptado por el TJCAN en el caso de Ecuador evidencia una mayor apertura para interpretar las excepciones al principio de libre circulación de mercancías. El TJCAN hizo una constatación más pragmática y acorde con el sentido del requisito de causalidad desarrollado en su jurisprudencia. El tribunal consideró que una de las causas de la emisión de GEI era el bióxido de carbono (CO2) emitido por los vehículos automotores (TJCAN, 2 de mayo de 2017, p. 33). A partir de esta constatación, el TJCAN concluyó que:

[...] en términos generales, limitar (cuantitativamente) el número de vehículos a importar, así como limitar (cuantitativamente) el número de CKD a importar para ensamblar vehículos, sí es una medida idónea para reducir GEI, o al menos evitar el incremento de GEI provenientes del parque automotor (TJCAN, 2 de mayo de 2017, p. 33).

El artículo 73 del Acuerdo de Cartagena exige que las medidas estén «destinadas a» proteger la salud y vida de las personas. La restricción cuantitativa adoptada por Ecuador estaba destinada a lograr este objetivo. En el marco de la OMC, el Órgano de Apelación ha empleado una evaluación cualitativa para analizar la contribución de la medida al logro del objetivo. Una evaluación cualitativa es suficiente para decidir sobre el significado de la contribución de la medida, no es necesario que se cuantifique la contribución de la medida para alcanzar el objetivo (Mavroidis, 2015, pp. 438, 439). Por ejemplo, en el caso Brasil-Neumáticos, el Grupo Especial optó por realizar un análisis cualitativo de la contribución de la prohibición a la importación al logro del objetivo perseguido (Dawar y Ronen, 2016, p. 10). La SGCAN adoptó un enfoque diferente, cuantificó la contribución de la medida al objetivo que se busca alcanzar tomando como base la emisión de GEI que generaba el sector transporte.

Sin embargo, los Grupos Especiales y el Órgano de Apelación han reconocido que solo revisan las medidas adoptadas y no los objetivos que se buscan alcanzar dado 
que los segundos son una facultad exclusiva de los Miembros de la OMC. A pesar de esto, se evidencia dos tendencias: por un lado, los Grupos Especiales y el Órgano de Apelación se muestran reacios a aceptar medidas drásticas como los embargos; y, por otro lado, cuanto más importante es el objetivo perseguido, más flexible será el estándar de revisión aplicado (Mavroidis, 2015, p. 436).

El TJCAN establece un criterio útil al adoptar medidas para proteger la salud y vida de las personas, aclarando que son los Países Miembros los que pueden diferenciar las medidas que deben adoptarse para alcanzar un objetivo. En palabras del TJCAN:

El hecho de que el sector transporte solo sea responsable del 3\% de la emisión de GEI no impide que Ecuador, ni ningún otro país de la CAN, adopte medidas de mitigación de GEI en dicho sector, por muy poco que sea su aporte en el total de GEI. Si un país busca mitigar (reducir o evitar el incremento) la emisión de GEI, puede implementar políticas diferentes para los diferentes sectores económicos, de manera que podrá establecer ciertas medidas para los sectores que generan la mayor cantidad de GEI y otras medidas para los sectores que emiten menos GEI. (TJCAN, 2 de mayo de 2017, p. 33)

Este razonamiento del TJCAN refleja el reconocimiento de la facultad de los Estados de decidir sobre sus políticas públicas, el grado de protección de la salud humana y la diferenciación que puede darse en las medidas a adoptar. Coincidentemente, en la OMC se ha reconocido esta facultad de los Estados. El Órgano de Apelación en el caso Brasil-Neumáticos sostuvo que: «el principio fundamental es el derecho que tienen los Miembros de la OMC de determinar el nivel de protección que consideren apropiado en cada situación» (OMC, 3 de diciembre de 2007, párr. 210). A pesar de estos avances en el seno de la OMC, también se ha discutido que en algunos casos los Miembros han tenido límites para introducir medidas sanitarias con el objetivo de proteger la salud humana. Uno de los casos que puso en evidencia este cuestionamiento fue el de Comunidad Europea-Hormonas donde la prohibición de importar carne y productos cárnicos tratados con hormonas fue considerada una violación del Acuerdo MSF. Esto provocó que se criticara a los órganos de solución de controversias y que se plantee el tema de la soberanía de los Estados (Fukunaga, 2016, pp. 534-535). Como ha sostenido Messenger (2016, p. 124) al referirse al Acuerdo MSF, la protección de la vida y salud de las personas no es materia de controversia; lo que está en debate es quien decide acerca de cuáles son los riesgos y sobre qué base se toma esa decisión. Corresponde a las instituciones andinas decidir con respecto a si una medida nacional cumple con las disposiciones en materia de excepciones al principio de libre circulación de mercancías.

Asimismo, se han dado argumentos con respecto a que los acuerdos comerciales merman la soberanía nacional. Las disputas en la OMC que involucran el análisis entre la liberalización del comercio y la protección de la salud han polarizado el 
debate entre los defensores de la salud y los propulsores del comercio internacional (Warikandwa y Osode, 2014, p. 1263). La participación de los Estados en los acuerdos comerciales conlleva una limitación de su capacidad para regular. Sin embargo, esa capacidad no puede ser innecesariamente limitada cuando está de por medio la protección de la salud humana. En otras áreas, a nivel multilateral en las negociaciones de la Ronda de Doha, los países en desarrollo insistieron en que se tenga en cuenta sus prioridades de desarrollo. Esto significaba, entre otras cosas, su derecho a continuar protegiendo sus industrias nacionales estratégicamente importantes a través de la aplicación de altos aranceles y proteger su espacio de política nacional en cuanto a las medidas de liberalización del comercio (Jones, 2015, p. 196). Si esto es así, la introducción de restricciones a las importaciones por motivos de salud también requiere el respeto por ese espacio.

El TJCAN aclaró que «el examen sobre la causalidad directa lo que busca determinar es si la medida restrictiva establecida por el Ecuador es idónea o no para mitigar los GEI de ese sector, independientemente de si ese sector es o no el principal generador del GEI» (TJCAN, 2 de mayo de 2017, pp. 33-34). El TJCAN hizo bien en no adoptar un enfoque cuantitativo al analizar la idoneidad de la medida en tanto tal enfoque desnaturaliza el objetivo de este requisito y, además, limita la capacidad de los Estados. Con esta jurisprudencia, el TJCAN marca una pauta a seguir en el sentido que no se va a considerar si el sector o actividad es el principal causante del problema para aceptar la idoneidad de la medida. Sin embargo, la sentencia del TJCAN deja algunas interrogantes. No queda clara la introducción de la terminología por parte del TJCAN. A partir de este caso, el TJCAN está requiriendo que «en términos generales» la medida sea idónea.

En la OMC al tratar el tema de la contribución de la restricción al comercio con el logro del objetivo, el Órgano de Apelación en el caso Brasil-Neumáticos determinó que: «Existe esa contribución cuando hay una relación auténtica de fines a medios entre el objetivo que se persigue y la medida en cuestión» (OMC, 3 de diciembre de 2007, párr. 210). El TJCAN exige que la medida adoptada por el País Miembro sea causa directa e inmediata de la reducción de GEI. En realidad, lo que se va analizar es si la medida restrictiva contribuye o no a dicha reducción. La pregunta que surge es si cualquier contribución al logro del objetivo, por mínima o marginal que sea basta para probar la relación de causalidad. La sentencia del TJCAN refleja que este órgano jurisdiccional está dispuesto a aceptar que se cumple con el requisito de la idoneidad si la medida contribuye de alguna forma con el objetivo buscado, independientemente del grado de contribución. En estos casos, debe considerarse la transcendencia del valor protegido. Con respecto al sector transporte y la emisión de GEI, el TJCAN ha aceptado la idoneidad «por muy poco que sea su aporte en el total de GEI». El criterio 
de «muy poco» abre la puerta para que se adopten una variedad de medidas restrictivas del comercio invocando el artículo 73 del Acuerdo de Cartagena.

3. El enfoque de la Secretaría General de la Comunidad Andina frente a las interpretaciones de los Grupos Especiales y el Órgano de Apelación en la Organización Mundial del Comercio

3.1. La protección de la salud de las personas como un interés vital en las interpretaciones de los Grupos Especiales y el Órgano de Apelación en la Organización Mundial del Comercio

En la CAN, para calificar una medida como restricción al comercio contraria al artículo 72 del Acuerdo de Cartagena, la SGCAN ha sostenido que se requiere analizar «los efectos eventualmente limitantes o restrictivos del comercio». Para ello, la SGCAN ha sostenido que de esta manera se ha entendido en la OMC y en la Comisión de la Unión Europea (SGCAN, 18 de junio de 1999, p. 5). No obstante, para analizar cuando una medida nacional puede ser justificada como excepción al principio de libre circulación de mercancías, en el caso Ecuador, la SGCAN adopta una posición que contrasta con la interpretación en la OMC.

En la OMC, el artículo XI.1 del GATT se dirige a las medidas en frontera y se dio no porque existiera un elemento de discriminación entre productos nacionales y extranjeros, sino porque se consideró que este tipo de medidas impedía el comercio internacional de mercancías como tal (Schütze, 2017, p. 45). Además, como sostiene Schütze (2017, p. 46), el alcance del término restricción es muy amplio y las medidas nacionales solo tienen que indirecta o potencialmente impedir o restringir el comercio para ser consideradas ilegales. Matsushita, Schoenbaum y Mavroidis (2015, p. 240) señalan que esta norma limita los derechos soberanos de los Estados para adoptar medidas con el fin de influir, limitar o bloquear la circulación del comercio a través de las fronteras.

Sobre la trascendencia del artículo XI.1 del GATT, el Grupo Especial en el caso Turquía-Textiles destacó que "es una de las piedras angulares del sistema del GATT» (OMC, 31 de mayo de 1999, párr. 9.63) y en Estados Unidos-Aves de Corral enfatizó que esta norma contiene «uno de los principios fundamentales del sistema jurídico del GATT/OMC» (OMC, 29 de septiembre de 2010, párr. 7.447).

A pesar de la relevancia del artículo XI del GATT, en los casos Estados UnidosCigarrillos de clavo de olor (OMC, 2 de setiembre de 2011, párr. 7.347), BrasilNeumáticos recauchutados (OMC, 12 de junio de 2007, párr. 7.108) y Comunidades Europeas-Amianto, los Grupos Especiales y el Órgano de Apelación coincidieron en sus interpretaciones con respecto a las excepciones del artículo XI del GATT. 
En estos casos, se destacó la importancia de la protección de la salud humana. En Comunidades Europeas-Amianto, el Órgano de Apelación reitera el criterio expresado en el caso Corea-Carne vacuna y señaló que: «Cuanto más vitales o importantes sean esos intereses o valores comunes», "más fácil será aceptar como "necesarias» unas medidas encaminadas a lograr esos objetivos» (OMC, 12 de marzo de 2001, párr. 172). En el caso de proteger la vida y la salud de las personas, el Órgano de Apelación marca la pauta seguida por los Grupos Especiales en el sentido que «El objetivo perseguido es vital y de la máxima importancia» (OMC, 12 de marzo de 2001, párr. 172). Este mismo enfoque ha sido expresado en el caso Indonesia-Carne de pollo (OMC, 17 de octubre de 2017, párr. 7.225). Desde el caso Estados UnidosCamarones, el Órgano de Apelación puntualizó que las excepciones previstas en el artículo XX del GATT han sido introducidas porque «se ha reconocido un carácter importante y legítimo a la política interna implícita en esas medidas» (OMC, 2 de octubre de 1998, párr. 121).

Las interpretaciones en la OMC tienden a centrarse en la protección a la salud como un derecho y deber de los Estados más que como un derecho humano de los ciudadanos debido a las controversias entre los Miembros sobre cómo interpretar los derechos humanos y económicos (Petersmann, 2016, p. 122). Los países en desarrollo han mostrado resistencia con respecto a la inclusión de una cláusula de derechos humanos en los Acuerdos de la OMC porque este tipo de inclusiones es vista como una forma de introducir políticas neocolonialistas y un medio a través del cual los países desarrollados pueden introducir barreras al comercio (Kanade, 2012, p. 390).

No obstante, el Órgano de Apelación ha mostrado su capacidad para leer el artículo XX del GATT de manera amplia. En especial, el significado de algunos términos de esta norma ha mostrado evolución (Harris y Moon, 2015, p. 21). Los casos más recientes muestran que los Grupos Especiales y el Órgano de Apelación han interpretado de manera amplia especialmente la justificación del artículo XX del GATT para proteger la vida y salud de las personas y la conservación de recursos naturales agotables (Weber, 2015, p. 417). Las interpretaciones del Órgano de Apelación, sin embargo, han establecido que los Grupos Especiales no deberían ser igualmente flexibles a los objetivos invocados por los Miembros de la OMC. El Órgano de Apelación ha aclarado que deberían ser más flexibles cuando la vida y salud humana están de por medio y menos cuando los Miembros buscan otros objetivos de política previstos en el artículo XX del GATT. Este entendimiento se da a pesar que el artículo XX del GATT no ha contemplado una jerarquía entre objetivos (Mavroidis, 2015, pp. 440-441). Así, en el caso China-Tierras Raras, China invocó el artículo $\mathrm{XX}$ literal b) y g) del GATT para justificar las restricciones a las exportaciones. Sin embargo, el Grupo Especial consideró que las consideraciones medioambientales en 
el caso se enfocaban más en el artículo XX literal g). Como resultado, China tuvo que asumir una mayor carga probatoria para persuadir sobre la justificación de sus medidas dado que hay más flexibilidad cuando se trata del artículo XX literal b) del GATT y el tema de la salud humana (Gao, 2017, p. 125).

Aun cuando no se ha establecido una jerarquía entre objetivos en el artículo XX del GATT y en el artículo 73 del Acuerdo de Cartagena, lo cierto es que el valor más importante es la vida y salud humana. Así también lo ha entendido el TJUE. En el caso de Peijper, el TJUE sostuvo que «entre los bienes o intereses protegidos por el artículo 36, la salud y la vida de las personas ocupan el primer lugar» (TJUE, 1976, párr.15; TJUE, 1994, párr. 16).

Particularmente, en el tema del transporte, en el caso Brasil - Tributación, el Grupo Especial observó el incremento de las emisiones de $\mathrm{CO} 2$ en Brasil y recordó la interpretación del Órgano de Apelación que destaca la importancia de la protección de la vida y salud de las personas (OMC, 30 de agosto de 2017, párr.7.915). Sobre esta base, el Grupo Especial enfatizó que «el grado de importancia de los intereses perseguidos por el Brasil (es decir, aumentar la seguridad de los vehículos y reducir las emisiones de CO2) es elevado» (OMC, 30 de agosto de 2017, párr.7.916). El Órgano de Apelación siempre ha tenido en cuenta en la evaluación de la medida cuestionada «la «importancia relativa» de los intereses o valores promovidos» por la misma (OMC, 7 de abril de 2005, párr. 306). La Resolución de la SGCAN en el caso Ecuador muestra poca preocupación por estos intereses.

\subsection{El análisis de la contribución de la medida al logro del objetivo perseguido en las interpretaciones de la Organización Mundial del Comercio}

En la OMC se ha analizado la contribución de la medida al logro del objetivo perseguido para determinar la justificación de la restricción al comercio a la luz del artículo XX literal b) del GATT. En el caso Brasil-Neumáticos, el Órgano de Apelación no estuvo de acuerdo con Brasil en el sentido de que «una prohibición de las importaciones cuya contribución es marginal o insignificante puede, no obstante, considerarse necesaria porque el objetivo es reducir en la máxima medida posible la exposición a los riesgos» (OMC, 3 de diciembre de 2007, párr.150). Sin embargo, el Órgano de Apelación mostró flexibilidad en el análisis de los requisitos para aceptar una restricción al comercio y aclaró que esto no implicaba que «una prohibición de las importaciones, o cualquier otra medida que tenga efectos de restricción del comercio, cuya contribución no sea inmediatamente perceptible, no pueda estar justificada en virtud del apartado b) del artículo $\mathrm{XX}_{\text {» }}(\mathrm{OMC}, 3$ de diciembre de 2007, párr.151). De manera similar, en el caso Brasil-Neumáticos, la importancia de evaluar los efectos de mediano y largo plazo de una medida así como hasta qué 
punto dicha medida puede conducir a una reducción de la exposición a los riesgos fue resaltada (Ruozzi, 2014, p. 164). Por su parte, al interpretar el Acuerdo MSF y la evaluación del riesgo, el Órgano de Apelación muestra apertura para entender los requisitos exigidos para imponer medidas nacionales, sosteniendo que "Cuando múltiples factores pueden contribuir a un riesgo específico, el evaluador del riesgo no está obligado a diferenciar la contribución individual de cada factor» (OMC, 16 de octubre de 2008, párr. 562). Este examen al producirse una multiplicidad de causas que producen el riesgo que busca encontrar un balance entre la libertad del evaluador del riesgo y el carácter científico de la evaluación del riesgo presta una considerable atención a uno de los temas más controvertidos en las disputas en el caso de la salud y medidas medioambientales (Ruozzi, 2014, p. 170). Esta apertura mostrada difiere de la posición de la SGCAN que cuestionó la idoneidad de la medida ecuatoriana porque solo se dirigía a un sector que contribuía con un 3\% en la emisión de GEI y existían otros factores de mayor contribución.

La interpretación desarrollada en el caso Brasil-Neumáticos no descarta la necesidad de adoptar medidas integrales y conjuntas para lidiar con los problemas a la salud. A partir de esta consideración, el Órgano de Apelación concluyó que:

[...] un grupo especial puede llegar a la conclusión de que una prohibición de las importaciones es necesaria sobre la base de la demostración de que la prohibición de las importaciones en litigio es adecuada para hacer una contribución importante al logro de su objetivo. (OMC, 3 de diciembre de 2007, párr. 151)

Este mismo enfoque fue planteado por el Grupo Especial en el caso China - Materias Primas. El Grupo Especial consideró que, según las interpretaciones del Órgano de Apelación, se pueden presentar dos tipos de contribuciones «la medida que «hace» una contribución importante al logro de su objetivo; y la medida que «es adecuada para hacer» una contribución importante al objetivo perseguido» (OMC, 5 de julio de 2011, párr. 7.484). Mostrando una mayor apertura para aceptar restricciones al comercio por razones de salud humana al analizar la relación entre la medida y el objetivo, se han aceptado medidas aun cuando su contribución «no sea inmediatamente perceptible» (OMC, 5 de julio de 2011, párr. 7.485).

Más recientemente, las interpretaciones en la OMC muestran una mayor flexibilidad en el sentido que una medida no tiene por qué estar dirigida a los sectores principales causantes del problema que se busca solucionar. En el caso Unión Europea-Focas, el Órgano de Apelación coincidió con el Grupo Especial al señalar que «el régimen de la UE [Unión Europea] para las focas "puede contribuir y contribuye" a su objetivo, o que lo hace "en cierta medida” (OMC, 22 de mayo de 2014, párr.5.289). Este enfoque es consistente con el estándar adoptado por los Grupos Especiales y el Órgano de Apelación en el sentido de que son los Miembros de la OMC los que 
definen el nivel de protección que se busca alcanzar. En el caso Brasil-Tributación, al evaluar la contribución de la medida brasileña al objetivo de lograr la seguridad de los vehículos y la reducción de la emisión de CO2, el Grupo Especial tuvo en cuenta el diseño, la estructura y el funcionamiento de los aspectos del programa INOVARAUTO. A partir de esta revisión, el Grupo Especial opinó que "las medidas que discriminan contra los productos importados no parecen hacer contribución alguna» (OMC, 30 de agosto de 2017, párr. 7.920). Entonces, sí se analiza la contribución de la medida al logro del objetivo; sin embargo, no se ponen límites con respecto a cuánto debe ser esa contribución.

Si las restricciones cuantitativas adoptadas por Ecuador en el sector transporte contribuyen al objetivo de lograr la reducción de la emisión de GEI o lo hace «en cierta medida" o hacen "contribución alguna" para que no aumenten dichas emisiones pueden ser consideradas como idóneas para alcanzar la protección de la salud humana.

Al referirse a las excepciones del artículo XX del GATT, Mitchell y Sheargold (2015, p. 151) han planteado que, debido a su naturaleza instrumental, su alcance no es claro y si se aplica o no a una medida nacional va a depender de cómo un tribunal interpreta la cláusula y la aplica a los hechos del caso. Así, concluyen que estas excepciones no proporcionan seguridad a los Estados con respecto, por ejemplo, al cumplimiento del tratado a través de sus medidas de control del tabaco. Lo mismo sucede con la aplicación del artículo 73 literal d) del Acuerdo de Cartagena. Por lo tanto, uno de los retos de la SGCAN y el TJCAN es determinar de qué manera van a lidiar con los casos en los cuales los Países Miembros adopten medidas medioambientales, en particular para proteger la salud. Consideramos que las instituciones andinas no deben dejar de tener en cuenta los intereses legítimos de los Estados en proteger valores no comerciales. Las batallas legales en materia de restricciones al comercio que involucren la protección de la salud deben ser consideradas con especial cuidado.

La adecuada protección de la salud humana por parte de los Estados va a depender de la capacidad de adaptación y evolución en las decisiones e interpretaciones de la SGCAN y el TJCAN para entender el derecho y el deber de los Estados de proteger este derecho humano. Este entendimiento debe estar presente aun cuando el artículo 73 del Acuerdo de Cartagena no se refiere específicamente a los derechos humanos, de lo contrario, las interpretaciones y decisiones de las instituciones andinas podrían convertirse en un problema para su realización.

En reiteradas oportunidades, el TJCAN ha expresado la necesidad de mantener y defender el principio de libre circulación de mercancías (TJCAN, 27 de agosto de 1997, p. 16), y ha enfatizado la importancia de eliminar los obstáculos al comercio para la integración económica y el rol central que juega este principio para alcanzar 
el mercado común (TJCAN, 29 de abril de 1997, pp. 15-16). Similarmente, la SGCAN ha sostenido que «los Países Miembros deben adoptar medidas que apunten hacia la liberación del intercambio comercial» (SGCAN, 6 de setiembre de 2002, p. 3). No obstante, ambas instituciones deben, a su vez, tener el debido cuidado en el tema de la protección de la salud consistente con el compromiso de los Estados expresado en la Carta Andina para la Promoción y Protección de los Derechos Humanos para lograr el «más alto nivel posible de salud física y mental» en la región andina. Un enfoque sesgado que se centra en el libre comercio y descuida objetivos vitales como la salud pública, como la decisión de la SGCAN en el caso de Ecuador analizado, es incongruente con esta necesidad y compromiso de los Estados.

\section{Conclusión}

A pesar de la flexibilidad que otorga el Acuerdo de Cartagena para que los Estados puedan tener un espacio de política y proteger, entre otros intereses, la vida y salud de las personas, el caso de Ecuador y la emisión de GEI muestra de qué manera la SGCAN dificulta y limita la utilización de ese espacio. Las excepciones previstas en el Acuerdo de Cartagena, si bien no deben ser objeto de abuso por parte de los Países Miembros para introducir medidas proteccionistas, han sido incorporadas para evitar un sistema rígido que ponga en el máximo nivel el libre comercio de mercancías sin respetar otros intereses vitales. Los Países Miembros tienen el derecho de adoptar la legislación que les permita proteger esos intereses de conformidad con lo establecido por el artículo 73 del Acuerdo de Cartagena. Un enfoque que no tenga en cuenta la contribución de una medida al logro de la protección de la salud humana contradice los objetivos del Acuerdo de Cartagena delineados en el artículo 1. Un enfoque cualitativo es necesario. Si bien el TJCAN rechazó el argumento de la SGCAN con respecto a la idoneidad de la medida ecuatoriana, la sentencia del TJCAN deja algunas interrogantes con respecto a si cualquier contribución de la medida al logro del objetivo, por mínima o marginal que sea basta para probar este requisito o será suficiente que «en términos generales» la medida sea idónea.

\section{Referencias bibliográficas}

Armingeon, K., Milewicz, K., Peter, S. y Peters, A. (2011). The constitutionalisation of international trade law (pp. 69-102). En T. Cottier y P. Delimatsis (eds.), The prospects of international trade regulation: From fragmentation to coherence. Cambridge: Cambridge University Press. https://doi.org/10.1017/CBO9780511792496.003

Dawar, K. y Ronen, E. (2016). How Necessary: A Comparison of Legal and Economic Assessments-GATT Dispute Settlements under Article XX (B), TBT 2.2 and SPS 
5.6. Trade, Law and Development, 8, 1.Recuperado de https://www.researchgate. net/publication/270648427_How_'Necessary'_A_Comparison_of_Legal_and_ Economic_Assessments_Under_GATT_Dispute_Settlements_Article_XXb_ TBT_22_and_SPS_56

Fukunaga Y. (2016). A Managerial Approach to Secure Compliance with the SPS Agreement. En J. Chaisse y T. Lin. International Economic Law and Governance Essays in Honour of Mitsuo Matsushita. Oxford: Oxford University Press. https://doi.org/10.1093/acpr of:oso/9780198778257.003.0031

Gao, F. (2017). WTO Trade and Environment Standard Rendering China-Rare Earths GATT Article XX Exemptions Impossible and Other International Laws Incompatible. Denver Journal of International Law and Policy, 45(1). Recuperado de https://papers. ssrn.com/sol3/papers.cfm?abstract_id=2913016

Harris, R. y Moon, G. (2015). GATT Article XX and Human Rights: What Do We Know from the First 20 Years? Melbourne Journal of International Law, 16(2), 1-52. Recuperado de https://law.unimelb.edu.au/_data/assets/pdf_file/0007/1687786/ Harris-and-Moon.pdf

Howse, R. (2012). Regulatory Measures. En A. Narlikar, M. Daunton, R. Stern y R.M. Stern (eds.), The Oxford Handbook on the World Trade Organization. Oxford: Oxford University Press.

IPCC - Intergovernmental Panel on Climate Change (2014). Summary for policymakers. En C.B. Field, V.R. Barros, D.J. Dokken, K.J. Mach, M.D. Mastrandrea, T.E. Bilir, M. Chatterjee, K.L. Ebi y.O. Estrada, R.C. Genova, B. Girma, E.S. Kissel, A.N. Levy, S. MacCracken, P.R. Mastrandrea y L.L. White (eds.), Climate Change 2014: Impacts, Adaptation, and Vulnerability. Part A: Global and Sectoral Aspects. Contribution of Working Group II to the Fifth Assessment Report of the Intergovernmental Panel on Climate Change. Cambridge: Cambridge University Press. Recuperado de https:// www.ipcc.ch/pdf/assessment-report/ar5/wg2/ar5_wgII_spm_en.pdf

Jones, K. (2015). Reconstructing the World Trade Organization for the 21st century: an institutional approach. Oxford: Oxford University Press. https://doi.org/10.1093/ acprof:oso/9780199366040.001.0001

Kanade, M. (2012). Human Rights and Multilateral Trade: A Pragmatic Approach to Understanding the Linkages. Journal Jurisprudence, 15, 389-418.

Kapterian, G. (2010). A Critique of the WTO Jurisprudence on 'Necessity'. International \& Comparative Law Quarterly, 59(1), 89-127. https://doi.org/10.1017/ S0020589309990091

Matsushita, M., Schoenbaum, T.J. y Mavroidis, P.C. (2015). The World Trade Organization. Law, Practice, and Policy. Oxford: Oxford University Press.

Mavroidis, P.C. (2015). The Regulation of International Trade: GATT. Massachusetts: MIT Press. https://doi.org/10.7551/mitpress/10465.001.0001

Messenger, G. (2016). The Development of World Trade Organization Law: Examining Change in International Law. Oxford: Oxford University Press. https://doi.org/10.1093/acpr of:oso/9780198716464.001.0001 
Mitchell, A. y Sheargold, E. (2015). Protecting the autonomy of states to enact tobacco control measures under trade and investment agreements. Tobacco control, 24(e2), e147-e153. https://doi.org/10.1136/tobaccocontrol-2014-051853

Organización Mundial del Comercio - OMC (2 de octubre de 1998). Informe del Órgano de Apelación. Estados Unidos — Prohibición de importar ciertos camarones y sus productos. Documento WT/DS58/AB/R.

Organización Mundial del Comercio - OMC (31 de mayo de 1999). Informe del Grupo Especial. Turquía - Restricciones a las importaciones de productos textiles y de vestido. Documento WT/DS34/R.

Organización Mundial del Comercio - OMC (12 de marzo de 2001). Informe del Órgano de Apelación. Comunidades Europeas - Medidas que afectan al amianto y a los productos que contienen amianto. Documento WT/DS135/AB/R.

Organización Mundial del Comercio - OMC (29 de mayo de 2002). Informe del Grupo Especial. Comunidades Europeas — Denominación comercial de sardinas, documento WT/DS231/R.

Organización Mundial del Comercio - OMC (7 de abril de 2005). Informe del Órgano de Apelación. Estados Unidos - Medidas que afectan al suministro transfronterizo de servicios de juegos de azar y apuestas. Documento WT/DS285/AB/R.

Organización Mundial del Comercio - OMC (12 de junio de 2007). Informe del Grupo Especial. Brasil - Medidas que afectan a las importaciones de neumáticos recauchutados. Documento WT/DS332/R.

Organización Mundial del Comercio - OMC (3 de diciembre de 2007). Informe del Órgano de Apelación. Brasil - Medidas que afectan a las importaciones de neumáticos recauchutados. Documento WT/DS332/AB/R.

Organización Mundial del Comercio - OMC (16 de octubre de 2008). Informe del Órgano de Apelación. Estados Unidos — Mantenimiento de la suspensión de obligaciones en la diferencia CE - Hormonas. Documento WT/DS320/AB/R.

Organización Mundial del Comercio - OMC (29 de septiembre de 2010). Informe del Grupo Especial. Estados Unidos - Determinadas medidas que afectan a las importaciones de aves de corral procedentes de China. Documento WT/DS392/R.

Organización Mundial del Comercio - OMC (5 de julio de 2011). Informe del Grupo Especial. China - Medidas relativas a la exportación de diversas materias primas. Documento WT/DS394/R, WT/DS395/R, WT/DS398/R.

Organización Mundial del Comercio - OMC (2 de setiembre de 2011). Informe del Grupo Especial. Estados Unidos - Medidas que afectan a la producción y venta de cigarrillos de clavo de olor. Documento WT/DS406/R.

Organización Mundial del Comercio - OMC (22 de febrero de 2012). Informes del Grupo Especial. China - Medidas relativas a la exportación de diversas materias primas, Documento WT/DS394/R / WT/DS395/R / WT/DS398/R y Corr.1.

Organización Mundial del Comercio - OMC (22 de mayo de 2014). Informe del Órgano de Apelación. Comunidades Europeas: Medidas que prohiben la importación y 
comercialización de productos derivados de las focas. Documento WT/DS400/AB/R, WT/DS401/AB/R.

Organización Mundial del Comercio - OMC (22 de diciembre de 2016). Informe del Grupo Especial. Indonesia - Importación de productos hortícolas, animales y productos del reino animal, Documento WT/DS477/R WT/DS478/R.

Organización Mundial del Comercio - OMC (30 de agosto de 2017). Informe del Grupo Especial. Brasil - Determinadas medidas relativas a la tributación y las cargas Informes del Grupo Especial. Documento WT/DS472/R.

Organización Mundial del Comercio - OMC (17 de octubre de 2017). Informe del Grupo Especial. Indonesia - Medidas relativas a la importación de carne de pollo y productos de pollo. Documento WT/DS484/R.

OMS - Organización Mundial de la Salud - OMS (1 de febrero de 2018). Cambio climático y salud. Notas descriptivas. Recuperado de http://www.who.int/es/news-room/ fact-sheets/detail/cambio-climático-y-salud

Petersmann, E.U. (2016). 20 Years WTO Law and Governance: Some Legal Methodology Problems. Manchester Journal of International Economic Law, 13(2), 106-127. Recuperado de http://hdl.handle.net/1814/44795

Reyes Tagle, Y. (2013). The Free Movement of Goods in Mercosur: Developing a European Court of Justice Approach in Mercosur? WTI Academic Cooperation Project Working Paper Series 2013/08. https://doi.org/10.2139/ssrn.2618408

Rincón Eizaga, L. (2013). Derechos humanos e integración en América Latina: El caso de la Comunidad Andina. Frónesis, 20(3), 411-433.

Ruozzi, E. (2014). The Multilateral Trading System and Non-Trade Issues: How the World Trade Organisation Managed to Integrate Environmental Concerns without Integrating International Environmental Law. En C. Herrmann, M. Krajewski y J.P. Terhechte. European Yearbook International Economic Law, 5 (pp. 141-171). Berlín: Springer. https://doi.org/10.1007/978-3-642-40913-4_7

Schütze, R. (2017). From international to federal market. The changing structure of European Law. Oxford: Oxford University Press. https://doi.org/10.1093/ oso/9780198803379.001.0001

Secretaría General de la Comunidad Andina - SGCAN (18 de junio de 1999). Resolución No 449. Gaceta Oficial del Acuerdo de Cartagena (G.O.A.C.), № 616.

Secretaría General de la Comunidad Andina - SGCAN (10 de setiembre de 1999). Resolución No 281. Gaceta Oficial del Acuerdo de Cartagena (G.O.A.C.), № 480.

Secretaría General de la Comunidad Andina - SGCAN (6 de setiembre de 2002). Resolución No 644. Gaceta Oficial del Acuerdo de Cartagena (G.O.A.C.), No 831.

Secretaría General de la Comunidad Andina - SGCANa (8 de mayo de 2003). Resolución No 724. Gaceta Oficial del Acuerdo de Cartagena (G.O.A.C.), No G.O.A.C. Nº 927.

Secretaría General de la Comunidad Andina - SGCAN (8 de febrero de 2005). Resolución No 897. Gaceta Oficial del Acuerdo de Cartagena (G.O.A.C.), No 1166. 
Secretaría General de la Comunidad Andina - SGCAN(18 de abril de 2013). Resolución N 1564. Gaceta Oficial del Acuerdo de Cartagena (G.O.A.C.), N 2181.

Secretaría General de la Comunidad Andina - SGCAN (18 de noviembre de 2013). Resolución N 1622. Gaceta Oficial del Acuerdo de Cartagena (G.O.A.C.).

Tribunal de Justicia de la Comunidad Andina-TJCAN (29 de abril de 1997). Proceso 3-AI96. Gaceta Oficial del Acuerdo de Cartagena (G.O.A.C.), No 261.

Tribunal de Justicia de la Comunidad Andina-TJCAN (27 de agosto de 1997). Proceso 2-AI96. Gaceta Oficial del Acuerdo de Cartagena (G.O.A.C.), № 289.

Tribunal de Justicia de la Comunidad Andina-TJCAN9 de marzo de 1998). Proceso 1-AN97. Gaceta Oficial del Acuerdo de Cartagena (G.O.A.C.), No 329.

Tribunal de Justicia de la Comunidad Andina-TJCAN (7 de agosto de 1998). Proceso 5-AN1997. Gaceta Oficial del Acuerdo de Cartagena (G.O.A.C.), No 361.

Tribunal de Justicia de la Comunidad Andina-TJCAN (20 de agosto de 1999). Proceso 28-IP-99. Gaceta Oficial del Acuerdo de Cartagena (G.O.A.C.).

Tribunal de Justicia de la Comunidad Andina-TJCAN (2 de agosto de 2000). Proceso 2-AN98. Gaceta Oficial del Acuerdo de Cartagena (G.O.A.C.), No 588.

Tribunal de Justicia de la Comunidad Andina-TJCAN (30 de noviembre de 2000). Proceso 1-AI-97. Gaceta Oficial del Acuerdo de Cartagena (G.O.A.C.), No 621.

Tribunal de Justicia de la Comunidad Andina-TJCAN (3 de junio de 2015). Proceso 183-IP2014. Gaceta Oficial del Acuerdo de Cartagena (G.O.A.C.), No 621. Recuperado de intranet.comunidadandina.org/documentos/Procesos/183-IP-2014.doc

Tribunal de Justicia de la Comunidad Andina-TJCAN (2 de mayo de 2017). Proceso 2-AN2015. Gaceta Oficial del Acuerdo de Cartagena (G.O.A.C.), No 3012.

Tribunal de Justicia de la Unión Europea - TJUE (1976). Asunto 104/75. Proceso penal contra Adriaan de Peijper, director de la sociedad Centrafarm BV. Recuperado de http://curia.europa.eu/juris/showPdf.jsf;jsessionid=9ea7d0f130d66a1f1b560a9848f 3a6b8f72e7e4d0754.e34KaxiLc3eQc40LaxqMbN4Pax8Le0?text=\&docid=89142\& pageIndex=0\&doclang $=E S \&$ mode $=1$ st $\&$ dir $=\&$ occ $=$ first $\&$ part $=1 \& \mathrm{cid}=122265$

Tribunal de Justicia de la Unión Europea - TJUE (1994). Asunto 320/93. Ortscheit v Eurim-Pharm.

Tribunal Permanente de Revisión del Mercosur (2005). Laudo $\mathrm{N}^{\circ} 1 / 2005$. Laudo del Tribunal Permanente de Revisión constituido para entender en el recurso de revisión presentado por la República Oriental del Uruguay contra el laudo arbitral del tribunal arbitral ad hoc de fecha 25 de octubre de 2005 en la controversia "prohibición de importación de neumáticos remoldeados procedentes del Uruguay». Asunción.

Van den Bossche, P. y Prévost, D. (2016). Essentials of WTO Law. Cambridge: Cambridge University Press. https://doi.org/10.1017/CBO9781139565448

Weber, R. (2015). Border Tax adjustment - legal perspective. Climatic Change, 133(3), 407-417. https://doi.org/10.1007/s10584-015-1414-2 
Wells, P.J. (2014). Unilateralism and protectionism in the World Trade Organization: The interpretation of the chapeau within GATT Article XX. Journal of International Trade Law \& Policy, 13(3), 222-231. https://doi.org/10.1108/JITLP-09-2013-0024

Warikandwa, T.V. y Osode, P.C. (2014). Managing the Trade-Public Health Linkage in Defence of Trade Liberalisation and National Sovereignty: An Appraisal of United States-Measures Affecting the Production and Sale of Clove Cigarettes. Potchefstroom Electronic Law Journal, 17(4), 1262-1285. https://doi.org/10.4314/pelj.v17i4.02

Recibido: 30 de marzo de 2019

Aprobado: 8 de agosto de 2019 